\title{
Robustness of the Prediction Filter in Differential Pulse Code Modulation System
}

\author{
Nikola B. Dankovic ${ }^{1}$, Zoran H. Peric ${ }^{2}$, Dragan S. Antic ${ }^{1}$, Stanisa Lj. Peric ${ }^{1}$, Sasa S. Nikolic ${ }^{1}$ \\ ${ }^{I}$ University of Nis, Faculty of Electronic Engineering, Department of Control Systems, \\ Aleksandra Medvedeva 14, Nis 18000, Republic of Serbia \\ ${ }^{2}$ University of Nis, Faculty of Electronic Engineering Department of Telecommunications, \\ Aleksandra Medvedeva 14, Nis 18000, Republic of Serbia \\ nikola.dankovic@elfak.ni.ac.rs
}

\begin{abstract}
Robustness of the differential pulse code modulation system with the first and second order predictor is considered in this paper. Special focus is on a robust stability of prediction filter with regard to predictor coefficients. Generalization of robustness in classical sense is performed and suitable relations for calculating probability of robustness are derived. The proposed method for robustness estimation is used for the first and second order prediction filters in the case of speech signal.
\end{abstract}

Index Terms-Gaussian distribution; probability density function; pulse modulation; robustness.

\section{INTRODUCTION}

Differential pulse code modulation (DPCM) is one of the most effective techniques for signal processing and transmission widely used in telecommunications, speech [1], [2] and image coding [3], [4], and medical research [5]-[7]. The transmission system based on DPCM is a nonlinear feedback system and as such is suitable for the analysis in control systems.

Linear prediction [8], [9], where the prediction of the current sample is calculated as the linear combination of the previous samples, is the basis of a DPCM system. Therefore a linear prediction (recursive) filter with predictor is, beside quantizer, the main part of each DPCM system. Sensitivity analysis for the prediction filter is given in [11], while stability analysis is performed for the first-order predictor [12] and second-order predictor [13].

Beside stability and sensitivity, robustness is also important property of each real system. An ability of a system to remain stable in the case of perturbations, system parameters variations, etc., is important system quality in control sense [14]. In the case of parametric robustness, the system is robust if it remains stable after perturbations in values of system parameters within certain boundaries [15].

Some robustness analyses of predictors in regard to transmission error have already done in [16]. In this paper we will perform parametric robustness in regard to predictor coefficients. First, we will give a classical robustness

Manuscript received 22 February, 2016; accepted 29 June, 2016.

This paper was supported by the Ministry for Education, Science and Technological Development of the Republic of Serbia (III 43007, III 44006, TR 35005). approach and, then, we will propose a generalization adopted for the real technical system with stochastic parameters.

Namely, in practice each system is in some way imperfect [17]-[21]. It means that system parameters are stochastic variables (not deterministic). In this case, classical methods for stability analysis are not applicable because we only know with which probability the system is robustly stable. In this paper, robustness probability estimation method is proposed and verified for the commonly used first-order and second-order predictor.

The rest of the paper is organized as follows. In Section II, DPCM system with linear predictor is described. Robustness analysis of prediction filter with experimental results for the first-order and second-order predictor is given in Section III. In Sections IV and V, we gave conclusions and possibility for further development in this area.

\section{DPCM SYSTEM - CONCEPT OF LINEAR PREDICTION}

The DPCM system, shown in Fig. 1, consists of encoder (quantizer, inverse quantizer and predictor) and decoder (inverse quantizer, predictor). The system is suitable for digitalization and transmission of highly correlated signals. This system quality is provided by a prediction filter in the feedback loop. The prediction filter estimates actual sample value based on one or more previous samples of input (source) signal. A number of previous samples, used for prediction, determines the predictor order, $k$ [1]
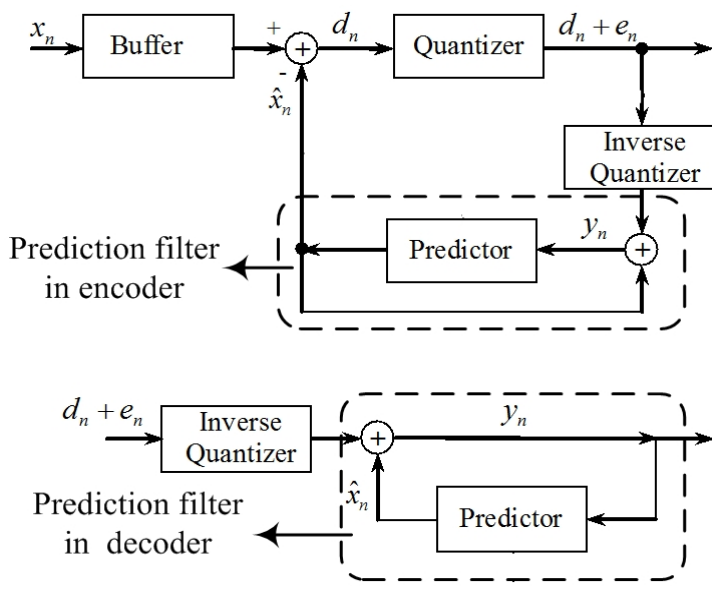

Fig. 1. Block scheme of DPCM system. 
The main concept of the DPCM system is to form the difference $d_{n}$ between the current sample $x_{n}$ and its predicted value $\hat{x}_{n}$. Quantization error $e_{n}$ occurs due to quantization of the difference $d_{n}$ (Fig. 1), [1], [7]. For the linear predictor, the predicted value $\hat{x}_{n}$ is calculated as a linear combination of the previously quantized reconstructed samples $y_{n-i}$. The functioning of the $k$-th order linear predictor is described with the following equation

$$
\hat{x}_{n}=\sum_{i=1}^{k} a_{i} y_{n-i}
$$

where $a_{i}, i=1,2, \ldots, k$ are the predictor coefficients.

DPCM systems are nonlinear feedback systems (quantizer is a nonlinear element). Robustness analysis of the whole DPCM system is very difficult. Quantizer has characteristic with saturation, i.e. it has limiter's behaviour [22]. It means that when input signal is increasing, quantizer gain is decreasing, which finally leads to better robust stability of the whole system. Hence, quantizer could have only positive effect on robust stability, i.e. robust stability of prediction filter is sufficient condition for robust stability of the whole system.

\section{The RobUSTNESS StABILITY OF THE LINEAR PREDICTION FILTER}

Analysis of prediction filter robustness is very important during the design of the system. It is well known that the predictor is a significant part of any DPCM/ADPCM (adaptive DPCM) system and its coefficients have a big effect on the system performances.

First, we will derive mathematical background for the robustness analysis of the prediction filter, in general.

Relation for $k$-th order predictor given by (1), in $z$ domain has the following form

$$
\hat{X}(z)=\left(\sum_{i=1}^{k} a_{i} z^{-i}\right) Y(z)=W_{P}(z) Y(z),
$$

where $W_{P}(z)=\left(\sum_{i=1}^{k} a_{i} z^{-i}\right)$ is the transfer function of the predictor.

Transfer functions of the prediction filter in the encoder and decoder, respectively, have the following forms:

$$
\begin{gathered}
W_{R}(z)=\frac{W_{P}(z)}{1-W_{P}(z)}=\frac{\sum_{i=1}^{k} a_{i} z^{-i}}{1-\sum_{i=1}^{k} a_{i} z^{-i}} \\
W_{R}^{D}(z)=\frac{1}{1-W_{P}(z)}=\frac{1}{1-\sum_{i=1}^{k} a_{i} z^{-i}} .
\end{gathered}
$$

Prediction filters are robustly stable if all the poles of the transfer functions (3) and (4) are inside the unit circle, i.e., if the characteristic equation: $1-\sum_{i=1}^{k} a_{i} z^{-i}=0$ (the same for the both filters) has all its zeroes inside the unit circle for all values of predictor coefficients. The previous characteristic equation can be written as

$$
z^{k}-\sum_{i=1}^{k} a_{i} z^{k-i}=0
$$

Stability conditions of the system described with characteristic equation (5) can be determined by several stability criterions [13]. Derivation of stability condition using Routh-Hurwitz criterion is given in Appendix A with special focus on the first- and second-order systems.

Now, we will consider robust stability of the prediction filter. We will use Kharitonov's theorem for estimation of parametric robustness [23].

The goal of this paper is to consider the robust stability of the prediction filter with commonly used first and second order predictor, although the proposed method is also applicable for higher order predictors. The first- and secondorder predictors are the most used in practice, because the prediction gain often gets into saturation for the higher orders [1], [2].

\section{A. Robustness of the First-Order Prediction Filter}

The characteristic equation (5) for the first order predictor has the following form:

$$
\left\{\begin{array}{l}
a_{0} z-a_{1}=0 \\
a_{0}=1
\end{array}\right.
$$

The set (family) of four characteristic polynomials using Kharitonov's theorem is:

$$
\left\{\begin{array}{l}
R_{1}(z)=\underline{a_{0}} z-\underline{a_{1}}, \\
R_{2}(z)=\overline{a_{0}} z-\underline{a_{1}}, \\
R_{3}(z)=\underline{a_{0}} z-\overline{a_{1}}, \\
R_{4}(z)=\overline{\overline{a_{0}}} z-\overline{a_{1}} .
\end{array}\right.
$$

We can notice that in this special case (for the first order), we actually have only two polynomials because $a_{0}=1$ :

$$
\left\{\begin{array}{l}
R_{1}(z)=z-\underline{a_{1}} \\
R_{2}(z)=z-\overline{a_{1}}
\end{array}\right.
$$

The prediction filter is stable if the roots of both polynomials are inside unit circle, i.e., $-1 \leq\left\{\underline{a_{1}}, \overline{a_{1}}\right\} \leq 1$.

Classical robustness analysis of the first-order prediction filter is finished because we suppose that predictor coefficients have exact values all the time, i.e. they are deterministic.

In practice, DPCM system and predictor as its part, are imperfect and predictor coefficients have stochastic parameters with normal distribution [17], [19], [20], [24]. 
We will estimate robust stability for the obtained values of predictor coefficients. We analyze recorded speech signal of 10200 samples with different frame lengths $M=10,20,50,100,150,200$ samples. For the obtained values of predictor coefficients $a_{1}$, we calculated mean values and standard deviations for all values of $M$ (Table I).

TABLE I. THE MEAN AND STANDARD DEVIATION OF THE FIRST ORDER PREDICTOR COEFFICIENT FOR DIFFERENT FRAME

\begin{tabular}{|c|c|c|c|c|c|c|}
\hline $\boldsymbol{M}$ [sample] & $\mathbf{1 0}$ & $\mathbf{2 0}$ & $\mathbf{5 0}$ & $\mathbf{1 0 0}$ & $\mathbf{1 5 0}$ & $\mathbf{2 0 0}$ \\
\hline $\boldsymbol{a}_{\mathbf{1 m}}$ & 0.803 & 0.874 & 0.917 & 0.928 & 0.932 & 0.929 \\
\hline $\boldsymbol{\sigma}_{\mathbf{1}}$ & 0.126 & 0.089 & 0.067 & 0.064 & 0.066 & 0.061 \\
\hline
\end{tabular}

From Table I, we can see that predictor coefficient has the maximal value for $M=150$ and minimal value for $M=10$, so $\overline{a_{1 m}}=0.932, \underline{a_{1 m}}=0.803$.

Appropriate probability density functions [19], [20] are:

$$
\begin{aligned}
& f\left(\overline{a_{1}}\right)=\frac{1}{\overline{\sigma_{1}} \sqrt{2 \pi}} \exp \left[-\frac{1}{2}\left(\frac{\overline{a_{1}}-\overline{a_{1 m}}}{\overline{\sigma_{1}}}\right)^{2}\right], \\
& f\left(\underline{a_{1}}\right)=\frac{1}{\underline{\sigma_{1}} \sqrt{2 \pi}} \exp \left[-\frac{1}{2}\left(\frac{\underline{a_{1}}-\underline{a_{1 m}}}{\underline{\sigma_{1}}}\right)^{2}\right],
\end{aligned}
$$

where $\overline{a_{1 m}}$ and $\overline{\sigma_{1}}$ are the mean value and standard deviation for maximal value of predictor coefficient $\overline{a_{1}}$, respectively; while $a_{1 m}$ and $\underline{\sigma_{1}}$ are the mean and standard deviation for minimal predictor coefficient $\underline{a_{1}}$, respectively. We should note that $\overline{\sigma_{1}}$ and $\underline{\sigma_{1}}$ are not maximal and minimal values of standard deviation.

Now, we can estimate robust stability as follows:

$$
\begin{aligned}
P_{1}= & \frac{\int_{-1}^{1} f\left(\overline{a_{1}}\right) d \overline{a_{1}}}{\int_{-\infty}^{\infty} f\left(\overline{a_{1}}\right) d \overline{a_{1}}}=\int_{-1}^{1} f\left(\overline{a_{1}}\right) d \overline{a_{1}}, \\
P_{2}= & \frac{\int_{-1}^{1} f\left(\underline{a_{1}}\right) d \underline{a_{1}}}{\int_{-\infty}^{\infty} f\left(\underline{a_{1}}\right) d \underline{a_{1}}}=\int_{-1}^{1} f\left(\underline{a_{1}}\right) d \underline{a_{1}} .
\end{aligned}
$$

Remark 2: Integrals in the denominators (11) and (12) present total probability and they are equal to 1 .

After solving integrals, we obtain the following values for robustness probability: $P_{1}=0.864$ and $P_{2}=0.941$.

The total robustness for the first-order prediction filter is: $P=P_{1} \times P_{2}=0.813$.

\section{B. Robustness of the Second-Order Prediction Filter}

Beside first-order predictors, the most common used predictors in DPCM systems are of the second-order. By using proposed method we can also estimate robustness for the prediction filter with the second-order predictor.

The characteristic equation (5) for the second-order predictor has the following form

$$
z^{2}-a_{1} z-a_{2}=0
$$

Now, the set of four characteristic polynomials is:

$$
\left\{\begin{array}{l}
R_{1}(z)=z^{2}-\underline{a_{1}} z-\underline{a_{2}} \\
R_{2}(z)=z^{2}-\overline{a_{1}} z-\underline{a_{2}} \\
R_{3}(z)=z^{2}-\underline{a_{1} z}-\overline{a_{2}} \\
R_{4}(z)=z^{2}-\overline{a_{1}} z-\overline{a_{2}}
\end{array}\right.
$$

The stability region, $S_{2}$, for the second-order prediction filter in parametric space $\left(a_{1}, a_{2}\right)$ is given by conditions, (see Appendix A):

$$
\left\{\begin{array}{l}
1+a_{1}-a_{2} \geq 0 \\
1-a_{1}-a_{2} \geq 0 \\
a_{2} \geq-1
\end{array}\right.
$$

Sufficient condition for robust stability is that pairs of coefficients in all four polynomials (7) satisfy inequalities (15). However, predictor coefficients are stochastic and we performed experiments for the same speech signal and also repeated experiment for the same values of frame length as in the case of the first-order predictor. Obtained values for the means and standard deviations of predictor coefficients are given in Table II.

TABLE II. THE MEAN AND STANDARD DEVIATION OF THE SECOND ORDER PREDICTOR COEFFICIENTS FOR DIFFERENT

\begin{tabular}{|c|c|c|c|c|c|c|}
\hline $\boldsymbol{M}$ [sample] & $\mathbf{1 0}$ & $\mathbf{2 0}$ & $\mathbf{5 0}$ & $\mathbf{1 0 0}$ & $\mathbf{1 5 0}$ & $\mathbf{2 0 0}$ \\
\hline $\boldsymbol{a}_{\boldsymbol{1} \boldsymbol{m}}$ & 0.988 & 1.131 & 1.288 & 1.385 & 1.431 & 1.438 \\
\hline $\boldsymbol{\sigma}_{\mathbf{1}}$ & 0.224 & 0.220 & 0.211 & 0.203 & 0.191 & 0.205 \\
\hline $\boldsymbol{a}_{\boldsymbol{2} \boldsymbol{m}}$ & -0.230 & -0.293 & -0.405 & -0.493 & -0.536 & -0.546 \\
\hline $\boldsymbol{\sigma}_{\mathbf{2}}$ & 0.197 & 0.211 & 0.211 & 0.204 & 0.180 & 0.192 \\
\hline
\end{tabular}

From Table II, we can see that predictor coefficient $a_{1}$ has the maximal value for $M=200$ and minimal value for $M=10$, predictor coefficient $a_{2}$ is maximal for $M=10$ and minimal for $M=200$, so: $\overline{a_{1 m}}=1.438, \underline{a_{1 m}}=0.988$, $\overline{a_{2 m}}=-0.230, \underline{a_{2 m}}=-0.546$.

Appropriate probability density functions for the polynomial $R_{1}$ is

$$
\begin{gathered}
f\left(\underline{a_{1}}, \underline{a_{2}}\right)=\frac{1}{\underline{\sigma_{1}} \sqrt{2 \pi}} \frac{1}{\underline{\sigma_{2}} \sqrt{2 \pi}} \times \\
\times \exp \left[-\frac{1}{2}\left(\frac{\underline{a_{1}}-\underline{a_{1 m}}}{\underline{\sigma_{1}}}\right)^{2}-\frac{1}{2}\left(\frac{\underline{a_{2}}-\underline{a_{2 m}}}{\underline{\sigma_{2}}}\right)^{2}\right],
\end{gathered}
$$


where $a_{1 m}$ and $\sigma_{1}$ are the mean value and standard deviation for minimal value of predictor coefficient $a_{1}: \underline{a_{1}}$, respectively; and $\underline{a_{2 m}}$ and $\underline{\sigma_{2}}$ are the mean and standard deviation for minimal value of predictor coefficient $a_{2}: \underline{a_{2}}$, respectively. In the same way, we form probability density functions for another three characteristic polynomials, $R_{2}: f\left(\overline{a_{1}}, \underline{a_{2}}\right), R_{3}: f\left(\underline{a_{1}}, \overline{a_{2}}\right)$ and $R_{4}: f\left(\overline{a_{1}}, \overline{a_{2}}\right)$.

Now, we can estimate robust stability. For $R_{1}$ we obtain

$$
P_{1}=\frac{\iint_{S_{2}} f\left(\underline{a_{1}}, \underline{a_{2}}\right) d \underline{a_{1}} d \underline{a_{2}}}{\int_{-\infty}^{\infty} \int_{-\infty}^{\infty} f\left(\underline{a_{1}}, \underline{a_{2}}\right) d \underline{a_{1}} d \underline{a_{2}}}=\iint_{S_{2}} f\left(\underline{a_{1}}, \underline{a_{2}}\right) d \underline{a_{1}} d \underline{a_{2}} .
$$

In the same way, robustness for other three characteristic polynomials are:

$$
\begin{aligned}
& P_{2}=\iint_{S_{2}} f\left(\overline{a_{1}}, \underline{a_{2}}\right) d \overline{a_{1}} d \underline{a_{2}}, \\
& P_{3}=\iint_{S_{2}} f\left(\underline{a_{1}}, \overline{a_{2}}\right) d \underline{a_{1}} d \overline{a_{2}}, \\
& P_{4}=\iint_{S_{2}} f\left(\overline{a_{1}}, \overline{a_{2}}\right) d \overline{a_{1}} d \overline{a_{2}},
\end{aligned}
$$

where $S_{2}$ is defined with (15).

We obtain the following values for robustness probability:

$$
\begin{aligned}
& P_{1}=0.962, \\
& P_{2}=0.641, \\
& P_{3}=0.791, \\
& P_{4}=0.232 .
\end{aligned}
$$

The total robustness for the second-order prediction filter is

$$
P=\prod_{i=1}^{4} P_{i}=0.113
$$

As we can see, the prediction filter is robustly stable with certain probability, unlike the ideal case when the predictor coefficients are perfectly adjusted. In this case total probability is low (25). The robustness probability decreases when the mean values of predictor coefficients, located inside stability region $S_{2}$, approach the bounds of region $S_{2}$. This decline is even more pronounced when the mean values of predictor coefficients, located outside stability region $S_{2}$, move away from region $S_{2}$, as in this case.

Remark 3: The robustness analysis presented above is the generalization of the classical robustness approach. In deterministic case $(\sigma=0)$ we obtain value of robustness probability equal to 1 (stable in the sense of robustness) or 0 (unstable). In the case of the first-order predictor probability of robustness is 1 , but in the case of the second-order predictor it is 0 because the values of predictor coefficients for $R_{4}$ do not satisfy conditions (15), so appropriate probability $P_{4}$ is low (24).

\section{CONCLUSIONS}

Robust stability analysis of the prediction filter with the most commonly used first- and second-order predictors was given in this paper. According to previously derived relations for stability region with respect to predictor coefficients, we proposed a method for determining robust stability of the prediction filter for concrete input signal and experimentally obtained values of predictor coefficients. Finally, we generalized the robustness analysis for a real system which is not perfect, i.e., when DPCM predictor coefficients are not perfectly adjusted (their values are normally distributed around the projected value). Robustness estimation was performed for the different frame lengths, and we calculated the probabilities which allow for the prediction filter to be robustly stable. This is a very important fact during the design of DPCM system. The proper selection of predictor coefficients could directly increase the system stability, leading, in that way, to even better performances of DPCM system.

\section{FUTURE WORK}

The proposed method for robustness estimation could be applied to prediction filter with higher order predictors, also. Kharitonov's theorem, used in this paper, is especially efficient in higher order systems for stability analysis. The problem which occurs is solving multidimensional integral needed for probability of robustness calculation. It is very difficult to determine the limits of integration area for higher order predictors. That is the reason why we would use Monte Carlo method for integration and try to obtain values for robustness probability with desired accuracy.

\section{APPENDIX A}

In order to obtain stability condition for the system described with (5), herein, we use Routh-Hurwitz stability criterion. The bilinear transformation $z=\frac{1+s}{1-s}$ is a mapping unit circle inside the $z$-plane into the left half of $s$-plane. After using the bilinear transformation onto (5), we obtain

$$
b_{k} s^{k}+b_{k-1} s^{k-1}+\ldots+b_{1} s+b_{0}=0
$$

where $b_{0}, b_{1}, \ldots, b_{k}$ are functions of predictor coefficients $a_{1}, a_{2}, \ldots, a_{k}$, i.e.: $b_{0}=\phi_{0}\left(a_{1}, \ldots, a_{k}\right), \ldots, b_{k}=\phi_{k}\left(a_{1}, \ldots, a_{k}\right)$. Applying the Hurwitz criterion, the stability region $S_{n}$ of difference equation (A1) is obtained. The system (5) is stable if all the zeroes of the characteristic equation (A1) are inside the left half of $s$-plane. We form Hurwitz determinant for (A1): 


$$
D_{k}=\left|\begin{array}{ccccc}
b_{k-1} & b_{k-3} & b_{k-5} & \cdots & 0 \\
b_{k} & b_{k-2} & b_{k-4} & \cdots & 0 \\
0 & b_{k-1} & b_{k-3} & \cdots & 0 \\
0 & b_{k} & b_{k-2} & \cdots & 0 \\
\vdots & \vdots & \vdots & \vdots & \vdots \\
0 & 0 & 0 & \cdots & b_{0}
\end{array}\right| .
$$

The necessary and sufficient condition for the stability of (5) is that all diagonal sub-determinants $D_{i}, i=1, \ldots, k$, are greater than zero:

$$
\left\{\begin{array}{l}
D_{1}=b_{k-1}>0, \\
D_{2}=\left|\begin{array}{cc}
b_{k-1} & b_{k-3} \\
b_{k} & b_{k-2}
\end{array}\right|>0, \\
\vdots \\
D_{k}>0 .
\end{array}\right.
$$

The stability region of (5), $S_{k}$, can be obtained by inequalities above for the first-order system up to $k$-th order system.

In the case of the first-order system, the stability region $S_{1}$ is given by the

$$
-1<a_{1}<1 .
$$

For the second-order system, the stability region $S_{2}$, in the parametric space $a_{1}, a_{2}$ is given by conditions:

$$
\left\{\begin{array}{l}
1+a_{1}-a_{2} \geq 0 \\
1-a_{1}-a_{2} \geq 0 \\
a_{2} \geq-1 .
\end{array}\right.
$$

\section{REFERENCES}

[1] N. S. Jayant, P. Noll, Digital Coding of Waveforms, Principles and Applications to Speech and Video. Prentice Hall, 1984, ch. 6.

[2] V. Despotovic, Z. Peric, L. Velimirovic, V. Delic, "DPCM with forward gain-adaptive quantizer and switched predictor for high quality speech signals", Advances in Electrical and Computer Engineering, vol. 10, no. 4, pp. 95-98, 2010. [Online]. Available: http://dx.doi.org/10.4316/aece.2010.04015

[3] W. Zschunke, "DPCM picture coding with adaptive prediction", IEEE Trans. on Communications, vol. 25, no. 11, pp. 1295-1302, 1977. [Online]. Available: http://dx.doi.org/10.1109/ TCOM.1977.1093771

[4] H. B. Mitchell, D. D. Estrakh, "A modified OWA operator and its use in lossless DPCM image compression", Int. Journal of Uncertainty, Fuzziness and Knowledge-Based Systems, vol. 5, no. 4, pp. 429-436, 1997 [Online]. Available: http://dx.doi.org/10.1142/S0218488597000324

[5] S. M. S. Jalaleddine, C. G. Hutchens, R. D. Strattan, W. Coberly, "ECG data compression techniques-a unified approach", IEEE Trans. on Biomedical Engineering, vol. 37, no. 4, pp. 329-343, 1990. [Online]. Available: http://dx.doi.org/10.1109/10.52340

[6] C. Fira, L. Goras, "An ECG signals compression method and its validation using NNs", IEEE Trans. on Biomedical Engineering, vol. 55, no. 4, pp. 1319-1326, 2008. [Online]. Available: http://dx.doi.org/10.1109/TBME.2008.918465

[7] A. V. Jocic, Z. H. Peric, M. R. Dincic, D. B. Denic, "Compression of the highly correlated measurement signals using DPCM technique", Elektronika ir Elektrotechnika, vol. 20, no. 4, pp. 76-79, 2014 [Online]. Available: http://dx.doi.org/10.5755/j01.eee.20.4.6889

[8] J. Benesty, M. Sondhy, Y Huang, Introduction to Speech Processing, in Springer handbook of speech processing, Eds. J. Benesty, M. Mohan Sondhi, and Y. A. Huan, Springer, 2008, ch. 7. [Online]. Available: http://dx.doi.org/10.1007/978-3-540-49127-9_7

[9] S. Paulikas, R. Karpavicijus, "Application of linear prediction coefficients interpolation in speech signal coding", Elektronika ir Elektrotechnika, vol. 80, no. 8, pp. 39-42, 2007. [Online]. Available: http://www.eejournal.ktu.lt/index.php/elt/article/view/11003

[10] J. Isailovic, "Sensitivity study and optimization of predictive systems for transmission of video signals", IEEE Trans. Aerospace and Electronic Systems, vol. 15, no. 1, pp. 156-161, 1979. [Online] Available: http://dx.doi.org/10.1109/TAES.1979.308808

[11] N. Dankovic, Z. Peric, D. Antic, D. Mitic, M. Spasic, "On the sensitivity of the recursive filter with arbitrary order predictor in DPCM system", Serbian Journal of Electrical Engineering, vol. 11, no. 4, pp. 609-616, 2014. [Online]. Available: http://dx.doi.org/10.2298/SJEE1404609D

[12] N. B. Dankovic, Z. H. Peric, "A probability of stability estimation of DPCM system with the first order predictor", Facta Universitatis, Series: Automatic Control and Robotics, vol. 12, no. 2, pp. 131-138, 2013.

[13] N. Dankovic, Z. Peric, D. Antic, A. Jocic, "Stability study of the second order recursive filter in DPCM system", Elektronika ir Elektrotechnika. (Submitted for publication)

[14] K. Zhou, J. C. Doyle, K. Glover, Robust Optimal Control. PrenticeHall, Englewood Cliffs, N.J., 1995. [Online]. Available: http://dx.doi.org/10.1016/0967-0661(96)83721-X

[15] M. Mansour, S. Balemi, W. Truol, Robustness of Dynamic Systems with Parameter Uncertainties. Birkhauser, Berlin, 1992. [Online]. Available: http://dx.doi.org/10.1007/978-3-0348-7268-3

[16] X. Song, T. Viero, Y. Neuvo, "Interframe DPCM with robust medianbased predictors for transmission of image sequences over noise channel", IEEE Trans. Image Processing, vol. 5, no. 1, pp. 16-25, 1996. [Online]. Available: http://dx.doi.org/10.1109/83.481667

[17] J. A. Borrie, Stochastic Systems for Engineers: Modeling, Estimation, and Control. Prentice Hall, 1992, New York.

[18] D. Antic, S. Nikolic, M. Milojkovic, N. Dankovic, Z. Jovanovic, S. Peric, "Sensitivity analysis of imperfect systems using almost orthogonal filters", Acta Polytechnica Hungarica, vol. 8, no. 6, pp. 79-94, 2011. [Online]. Available: http://www.uniobuda.hu/journal/Antic_Nikolic_Milojkovic_Dankovic_Jovanovic_P eric_32.pdf

[19] D. Antic, Z. Jovanovic, N. Dankovic, M. Spasic, S. Stankov, "Probability estimation of certain properties of the imperfect systems", in Proc. of IEEE 7th Int. Symposium on Applied Computational Intelligence and Informatics, (SACI 2012), Timisoara, Romania, 2012, pp. 213-216. [Online]. Available: http://dx.doi.org/10.1109/SACI.2012.6250004

[20] D. Antic, Z. Jovanovic, N. Dankovic, M. Spasic, S. Stankov, "Probability estimation of defined properties of the real technical systems with stochastic parameters", Scientific Bulletin of the "Politehnica" University of Timişoara, Romania, Trans on Automatic Control and Computer Science, vol. 57, no. 2, pp. 67-74, 2012.

[21] D. Antic, Z. Jovanovic, V. Nikolic, M. Milojkovic, S. Nikolic, N. Dankovic, "Modelling of cascade-connected systems using quasiorthogonal functions", Elektronika ir Elektrotechnika, vol. 18, no. 10 , pp. 3-8, 2012. [Online]. Available: http://dx.doi.org/10.5755/ j01.eee.18.10.3051

[22] S. Tarbouriech, G. Garcia, J. M. Gomes da Silva Jr, I. Queinnec, Stability and Stabilization of Linear Systems with Saturating Actuators. Springer, 2011. [Online]. Available: http://dx.doi.org/10.1007/978-0-85729-941-3

[23] V. L. Kharitonov, "Asymptotic stability of an equilibrium position of a family of systems of linear differential equations", Differensial 'nye Uravnenya, vol. 14, no. 11, pp. 2086-2088, 1978. (In Russian)

[24] L. Goldstein, B. Liu, "Deterministic and stochastic stability of adaptive differential pulse code modulation", IEEE Trans. Information Theory, vol. 23, no. 4, pp. 445-453, 1977. [Online] Available: http://dx.doi.org/10.1109/TIT.1977.1055754 